\title{
Significant reductions to frequent attenders to secondary services
}

\author{
Author: Karen Taylor
}

Aims

The aim of the project was to investigate if taking a different, more holistic patient-centred approach can identify hidden, complex care needs and alter admission rates for frequent attenders to secondary care services.

\section{Methods}

The project involved using an enhanced discharge coordinator located on the respiratory specialist wards. A group of high intensity service users were identified from hospital admission data and clinical referral. The care needs of these patients and factors such as disease severity, age, social deprivation and social isolation and comorbidities (including mental health) were assessed. The patients' admission data was plotted over time and reviewed to see if there were any trends or patterns that correlated to their service use. High-quality motivational interviewing conversations with patients were then undertaken and care needs were identified and addressed. This included a wide range of interventions such as enhanced cross service communications, addressing social isolation and unmet mental health need. The patients' secondary care service use was then measured post intervention.

\section{Results}

In total, the project identified 118 patients over 12 months who were high service users. In particular the service identified a group of patients who were initially identified as not requiring additional support from primary or secondary care but who were high intensity service users. As the project evolved the number of interventions available expanded. 85 patients were discussed with the general practitioner care coordinators (GPCC). GPCC were not aware that 58 patients were high intensity users. Seventeen were referred for cognitive behavioural therapy, 85 had one or more intervention recommended ranging from community matron intervention, benefit checks, referrals to occupational therapy and physiotherapy and more diverse interventions. Seven patients were tracked and the data shows that this holistic individualised approach resulted in a reduction in bed days of approximately over 700 days equating to a potential cost avoidance of up to $£ 210,000$

\section{Conclusion}

A change in approach to how high intensity service users are identified and managed while in and on discharge from secondary care can result in significant savings in terms of bed days. Using the new approach has resulted in reduced re-admissions and longer periods between admissions. This supports a more holistic approach to discharge planning for those with complex needs.

\section{Conflict of interest statement}

None 